\title{
OSA-Based Service Platform for All-IPv6 Network Environments
}

\author{
Yao-Chung Chang, Jiann-Liang Chen, Han-Chieh Chao, Senior Member, IEEE, and Sy-Yen Kuo, Fellow, IEEE
}

\begin{abstract}
The use of IP (Internet protocol) technology in the information and communications industry constitutes a major global trend. A highly efficient service architecture, enabling technologies and advanced applications are essential to rapid multimedia services in an all-IPv6 network environment. This work presents an all-IPv6 service platform based on open service architecture (OSA) to support a set of standard interfaces and applications. The all-IPv6 network environment was integrated using a network-processor-based IPv4/IPv6 translator and a mobile router (MR) supported IPv6 network mobility. The feasibility of the open service platform for all-IPv6 network environments and of the designed application programming interfaces was examined using three applications: e-commerce, video-on-demand, and on-line gaming. The performance analysis indicates that the system throughput increased from 10.5 to $60.5 \mathrm{Mb} / \mathrm{s}$ as the number of users increased from 1 to 80 ; the mean response time increased from approximately 1 to $10.5 \mathrm{~ms}$, and the delay time increased from 0.1 to $1 \mathrm{~ms}$.
\end{abstract}

Index Terms-All-IPv6 networks, application programming interface (API), IPv4/IPv6 translator, Java APIs for integrated networks (JAIN) platform, network mobility, open service architecture (OSA).

\section{INTRODUCTION}

$\mathbf{T}$ HE Internet protocol version 6 (IPv6) protocol is fundamental to the growth of Internet and has the potential to improve IP functionality and performance [1], [2]. IPv6 supports new Internet applications, and broadens technological development [3]. Corporations such as Microsoft and Nokia have published white papers on accelerating IPv6 development [4], [5]. Numerous new applications and Operating Systems, including Windows XP and Linux kernel 2.1.8 and higher, already incorporate IPv6 support, but some serious challenges remain in ensuring a successful and smooth transition from Internet protocol version 4 (IPv4) networks. IPv6-based networks have been implemented in isolation, but now the information technology (IT) industry seeks to connect these IPv6 islands across the IPv4 ocean using IPv4/IPv6 translators, and mobile

Manuscript received December 16, 2004, revised May 13, 2005. This work was supported in part by the National Dong Hwa University and in part by the National Taiwan University under the sponsorship of the National Science Council, R.O.C., under Project NSC 92-2219-E-259-003 and Project NSC 92-2219-E-002-020, and in part by the Taiwan NICI IPv6 Steering Committee, R\&D Division, under Contract R-0300.

Y.-C. Chang and J.-L. Chen are with the Department of Computer Science and Information Engineering, National Dong Hwa University, Hualien 974, Taiwan, R.O.C. (e-mail: changyc@mail.ndhu.edu.tw; lchen@mail.ndhu.edu.tw).

H.-C. Chao is with the Department of Electronic Engineering, National Ilan University, I-Lan 260, Taiwan, R.O.C. (e-mail: hcc@mail.ndhu.edu.tw).

S.-Y. Kuo is with the Department of Electrical Engineering, National Taiwan University, Taipei 106, Taiwan, R.O.C. (e-mail:sykuo@cc.ee.ntu.edu.tw).

Digital Object Identifier 10.1109/JSAC.2005.856823 routers (MRs) to support network mobility. When a moving vehicle connects to the Internet to obtain driving information, or an aircraft carries passengers who want to receive their e-mail, mobility mechanisms must be supported. Some studies have been published on Network Mobility (NEMO), including those of the Nautilus6 working group [6] and the IST OverDRiVE project [7]. The Nautilus6 working group is part of the WIDE organization and is managed by volunteers. Nautilus6 has implemented NEMO basic support on NetBSD 1.6.1 with a KAME MIP6. OverDRiVE (spectrum efficient unicast and multicast over dynamic radio networks in vehicular environments) is a research and technology development (RTD) project sponsored by the European Commission as part of the Information Technologies Program (IST). One project working group has specified a MR to provide multiradio access to a moving intravehicular area network (IVAN). Related studies include Connexion by Boeing [8] and InternetCAR in intelligent transportation systems (ITS) [9].

The development of a highly performing all-IPv6 network for information services will soon become an important issue. This work comprehensively reviewed domestic and international projects and trials associated with the development of all-IP networks [10], [11]. The development of high-quality all-IPv6 architecture must fulfill the following requirements.

1) Best-in-class telecommunication and data communication standards.

2) Improvement of existing specifications to yield an open system.

3) Smooth evolution of both networks and applications.

4) Highly reliable and efficient end-to-end QoS support.

5) Support for mobility and seamless handoff between accesses.

6) Separation of service, connection, and mobility control.

7) Rapid and flexible creation of new services

This work concerns the final two points, 6) and 7). An open service architecture (OSA) was constructed to accelerate the integration of telecommunications and data communications, and the ultimate goal is to develop high-quality information applications for the all-IPv6 network [12]. This OSA platform requires the following service characteristics: independence of platform, independence of application, multiple service support, service collaboration support, and support and simplicity of multiple network technology [13].

This paper is organized as follows. Section II introduces background of all-IPv6 network environments. Section III proposes an OSA-based service platform for all-IPv6 network environments of application programming interface (API) developments. Section IV describes the implementation of the service platform, including the IPv4/IPv6 translator based on 


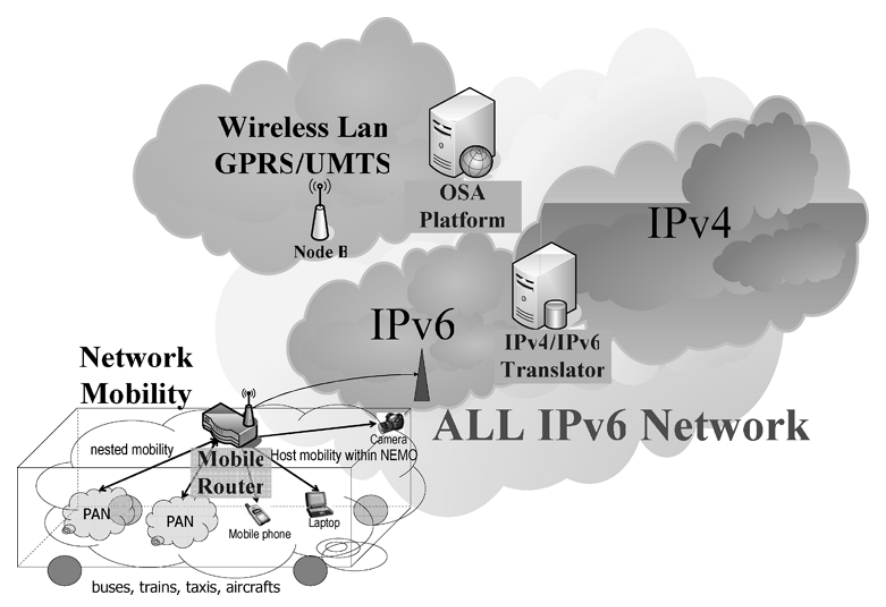

Fig. 1. All-IPv6 network environments.

the network processor and the MR supports network mobility. Section V presents three OSA-based application scenarios and analyzes the performance of OSA-based applications in all-IPv6 network environments. Section VI draws conclusions.

\section{All-IPv6 Network EnVIRONMENTS}

This work integrates the all-IPv6 network environment using an IPv4/IPv6 translator, based on a network processor, and a MR that supports IPv6 network mobility (Fig. 1). Therefore, users with IPv4 or IPv6 network connections, mobile equipments of the PAN or mobile can directly make use of benefits from the OSA-based applications. The following sections introduce IPv4/IPv6 transition and the network mobility.

\section{A. IPv4/IPv6 Transition}

The transition between today's IPv4 Internet and the IPv6based Internet of the future will be a long process, during which both protocols coexist. A mechanism for ensuring smooth, stepwise and independent changeover to IPv6 services is required. Such a mechanism must facilitate the seamless coexistence of IPv4 and IPv6 nodes during the transition period. Internet Engineering Task Force formed the Ngtrans Group to facilitate smooth transitions from IPv4 to IPv6 services. This work employs the IPv4/IPv6 translator mechanisms for all-IPv6 network environments to support applications in OSA.

The fundamental function of translation in IPv4/IPv6 transitioning is to translate IP packets. Numerous of translation mechanisms are based on the SIIT (stateless IP/ICMP translation algorithm) [14]. The SIIT algorithm is applied as the basis of network address translation-protocol translation (NAT-PT) mechanisms. The NAT-PT mechanism is a stateful IPv4/IPv6 translator [15]. NAT-PT nodes are at the boundary between IPv6 and IPv4 networks. Each node maintains a pool of globally routable IPv4 addresses, which are dynamically assigned to IPv6 nodes when sessions are initiated across the IPv6/IPv4 boundary. This arrangement allows native IPv6 nodes and applications to communicate with native IPv4 nodes and applications, and vice versa.

The NAT-PT translation architecture, presented in Fig. 2, also includes an application level gateways (ALG). The NAT-PT

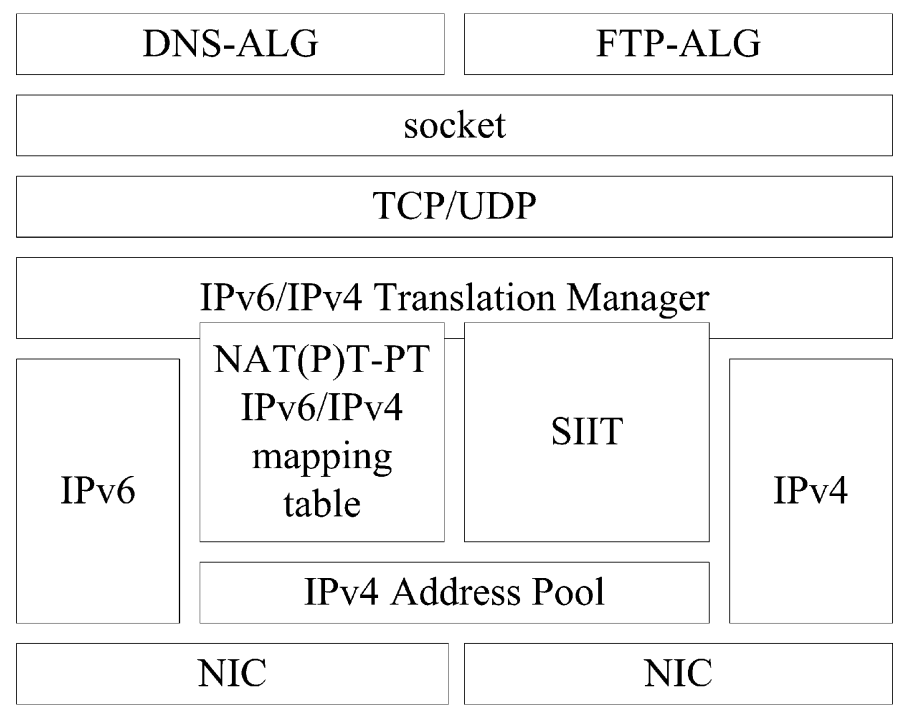

Fig. 2. Basic NAT-PT translation architecture.

mechanism does not snoop payloads, and the application may thus be unaware of it. Therefore, the NAT-PT mechanism depends on ALG agents that enable an IPv6 node to communicate with an IPv4 node and vice versa. The NAT-PT mechanism is an interoperability solution that requires no modification or extra software for use with either the IPv4 or the IPv6 network. The mechanism applies the required interoperability functions in a core network, making interoperability between nodes easier to manage and faster to manifest.

\section{B. Network Mobility}

The Mobile IPv6 network environment must support the link layer handoff and management mechanism to make network devices mobile. Mobile IP [16], [17] provides the mobility in the IP layer by two-tier addressing. This IP mobility support protocol comprises three functions: agent discovery, address binding update, and tunneling.

A group of nodes that move in a single direction at the same speed in a vehicular area network must be regarded as exhibiting network mobility, [18] as recently defined by the IETF working group, rather than host mobility. All nodes in the mobile network apply a special gateway called a MR, which handles the motion of the mobile network. Three network mobility solutions are HMIPv6 [19], prefix scope binding updates (PSBU) [20], and MRHA tunnel [21]. Each solution has particular strengths, but only the MRHA tunnel method is effective with NEMO. This work applies the MRHA tunnel method to the network architecture.

Hierarchical Mobile IPv6 (HMIPv6): The HMIPv6 protocol does not change the corresponding node's (CN's) operations. Changing these operations requires the incorporation of another network component, the mobile anchor point (MAP), into the protocol. The Mobile IPv6 protocol generates "bing update storms" whenever the mobile nodes (MNs) simultaneously process handoff operations. HMIPv6 represents a solution to this problem.

HMIPv6 utilizes a local home agent as the MAP. When MN moves in to a MAP's coverage area, MN yields the on-link 


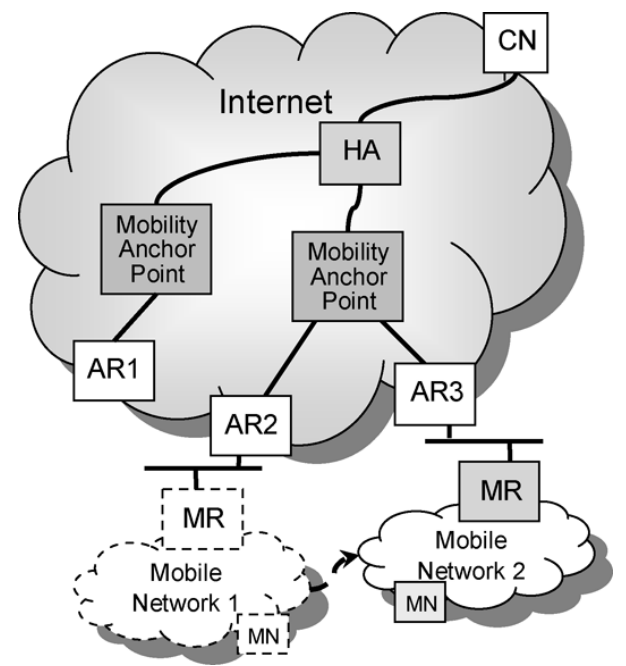

Fig. 3. Hierarchical Mobile IPv6.

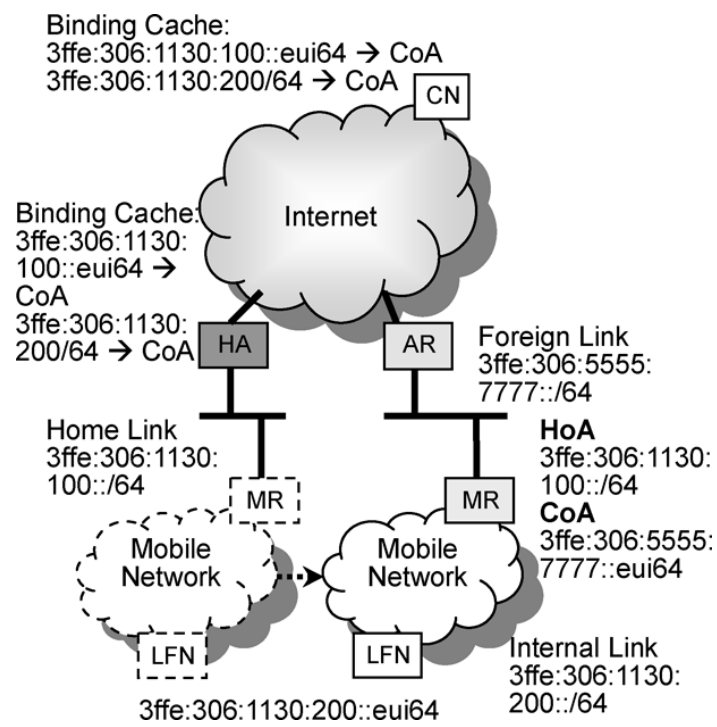

Fig. 4. Prefix scope binding update.

care-of-address (CoA) and sends binding update (BU) messages. When the MAP receives the BU message, it records the BU cache and sends the BU message to the home agent (HA) using the regional CoA of the MAP address. The MAP constructs the tunnel between the MN and the HA. MAP stores the routing information between regional $\mathrm{CoA}$ and on-link CoA. The $\mathrm{CN}$ sends its packets via the HA to the MAP, which transfers them to the MN in its coverage area. Hence, the MN updates the binding cache only when it moves into a different MAP, as shown in Fig. 3.

Prefix Scope Binding Updates (PSBUs): The PSBU method is also designed to prevent the "binding update storm" (Fig. 4) This protocol extends the traditional BU operations of MIPv6, MRs, HAs and CNs. The PSBU modifies the CoA of one prefix to perform the $\mathrm{BU}$ of numerous nodes connected as the mobility network.

The MR is prefixed with CoA when the mobile network moves into another area. It transmits BU messages that contain
- MRHA Tunnel

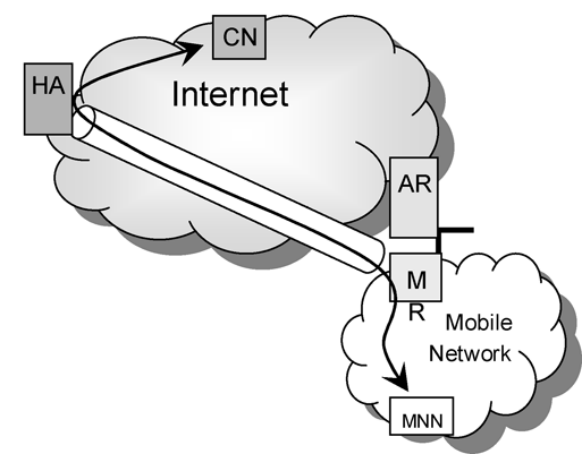

Fig. 5. MRHA tunnel.

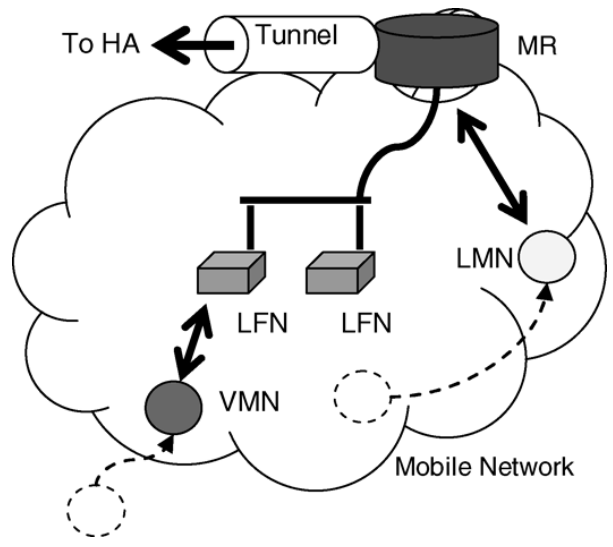

Fig. 6. Nodes within the mobile network.

CoA information to all MNs in the mobile network. The P-bit of the $\mathrm{BU}$ message is set to one to state that the $\mathrm{BU}$ message is a part of the PSBU mechanism. When the HA receives the BU messages, it checks the P-bit to identify the network prefix suboption field. The prefix information between the mobile network and the MR CoA are added to the biding cache. The $\mathrm{CN}$ checks the prefix address of the destination to specify the routers through which data are transmitted.

MRHA Tunnel: Fig. 5 depicts a mobile network that comprises three nodes, which may be fixed (LFN) or mobile (LMN, VMN), according to the classification of the IETF NEMO working group. A local fixed node (LFN) may be a host or a router, and is topologically fixed in relation to the MR, whereas a local mobile node (LMN), whose home link is in the mobile network, can adjust its attachment point in relation to the MR, as can the visiting mobile node (VMN) whose home link is not part of the mobile network.

Applying the MR to handle the movement of mobile network has two particular advantages. Nodes within the mobile network obtain prefixes from the MR. Additionally, the MR of the mobile network connects to external AR and maintains its private $\mathrm{MN}$ address. NEMO has three main strengths. First, it involves no direct connection between MN and AR. It also saves power by reducing the radio frequency. Finally, NEMO can support route optimization and the nested NEMO problem displayed in Figs. 6 and 7. 


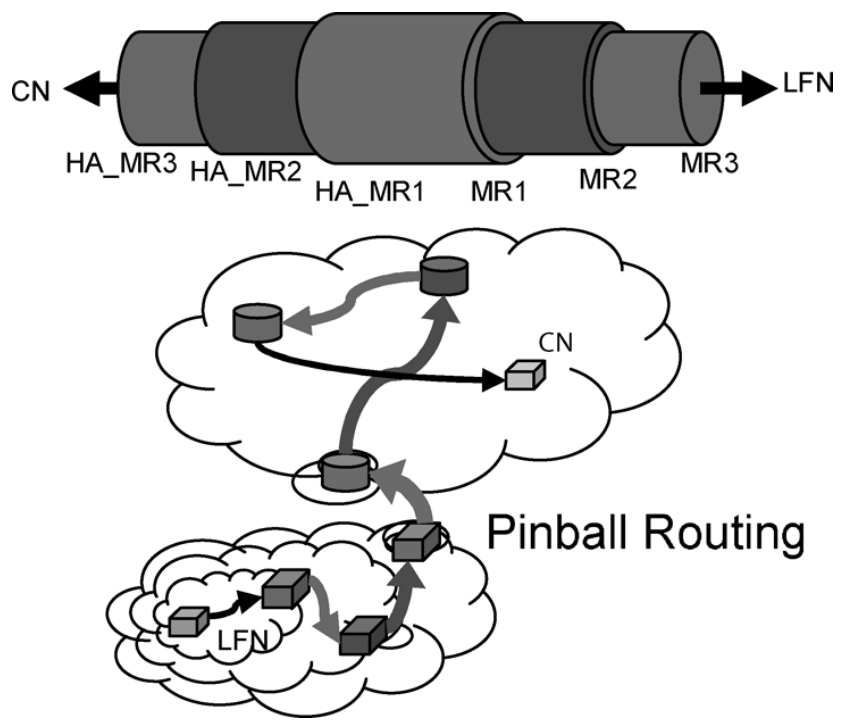

Fig. 7. Nested NEMO problem.

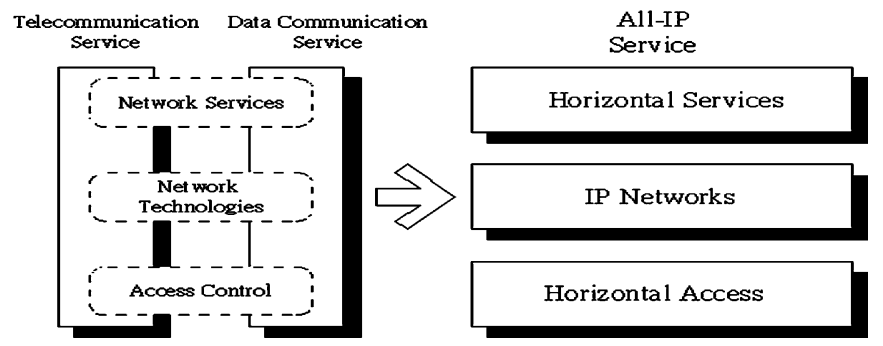

Fig. 8. Variation between vertical and horizontal service structure.

\section{OSA-BASEd SERVICE PLATFORM FOR ALl-IPv6 NETWORK ENVIRONMENTS}

Traditional telecommunication and data communication services are vertically integrated. Each service depends on a dedicated network and corresponding terminals. The all-IP concept changes the vertical structure into a horizontal one, as an overlay system presented in Fig. 8. A highly efficient service architecture, which enables various technologies and advanced applications, is critical in an all-IPv6 network environment, to provide high-quality services. This work addresses these requirements in an OSA service platform. This section summarizes important OSA-related knowledge and technology.

\section{A. Open Service Architecture (OSA)}

Open service architecture (OSA) has an open system structure, which bridges various systems [22]-[24]. That is, although each manufacturer's internal systems may differ, they all communicate with each other using the OSA platform. The OSA platform is an open construction proposed by 3GPP (3G Partnership Project), which provides the standard API to the operating system. Accordingly, Internet applications can conveniently exploit the network's service capacity via the API, as shown in Fig. 9. This open interface construction is sufficiently flexible and expandable to meet the demands of the network.

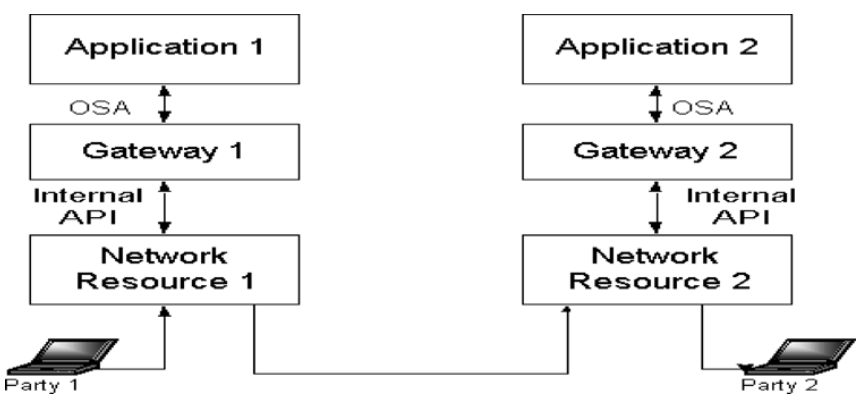

Fig. 9. OSA system application processes.

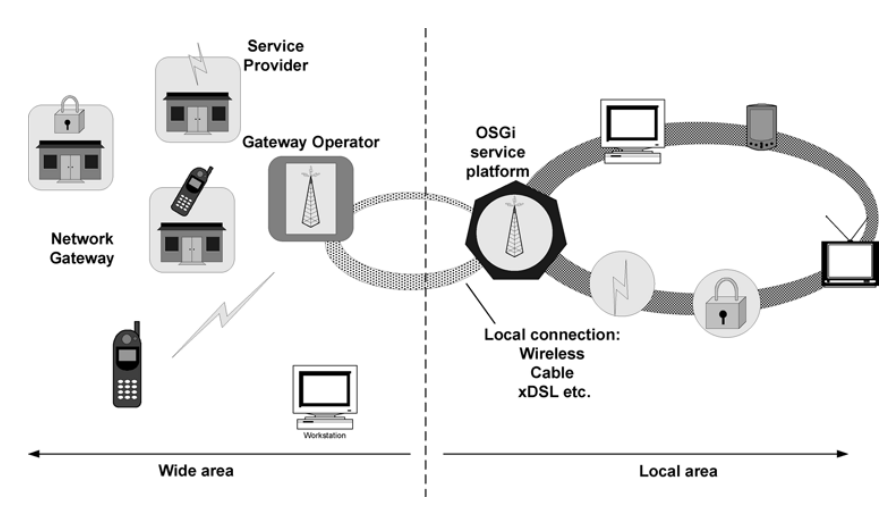

Fig. 10. OSGi concept.

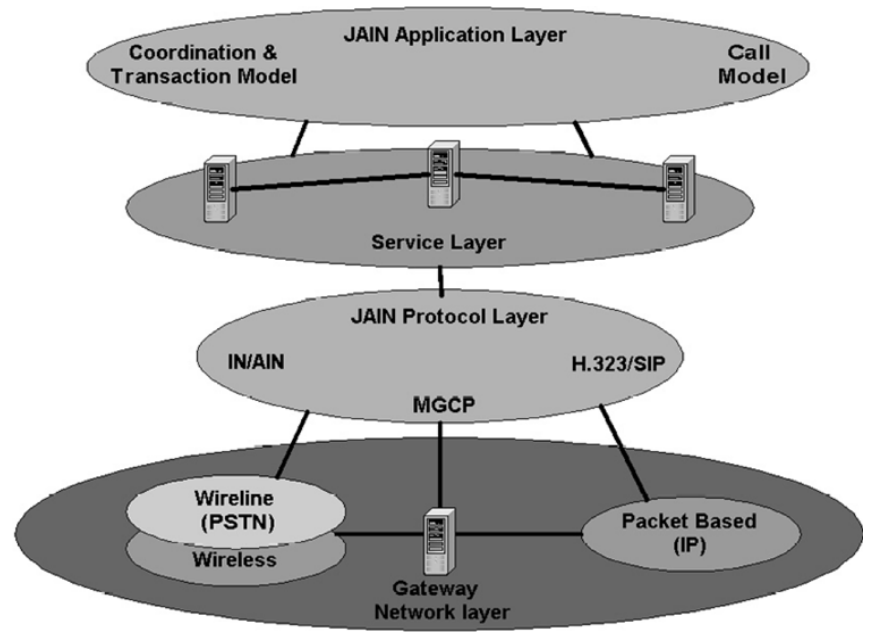

Fig. 11. JAIN platform.

\section{B. Open Services Gateway Initiative (OSGi)}

The OSGi organization was established in 1999, with the primary purpose of constructing an open standard interface to enable wide-area network services to communicate with home networking equipment. Fig. 10 depicts the OSGi concept [25]-[28]. Over 100 manufacturers already support OSGi. The OSGi 3.0 standard was announced in March 2003.

The OSGi standard, based on Java technology, enables service operators, manufacturers of household appliances, and Internet service providers to link with standard network interfaces. This standard consists of several substandards that use functions to distinguish between the structure of the application and resource management APIs; between the client and the wide-area network APIs; between the terminal and the local area network 


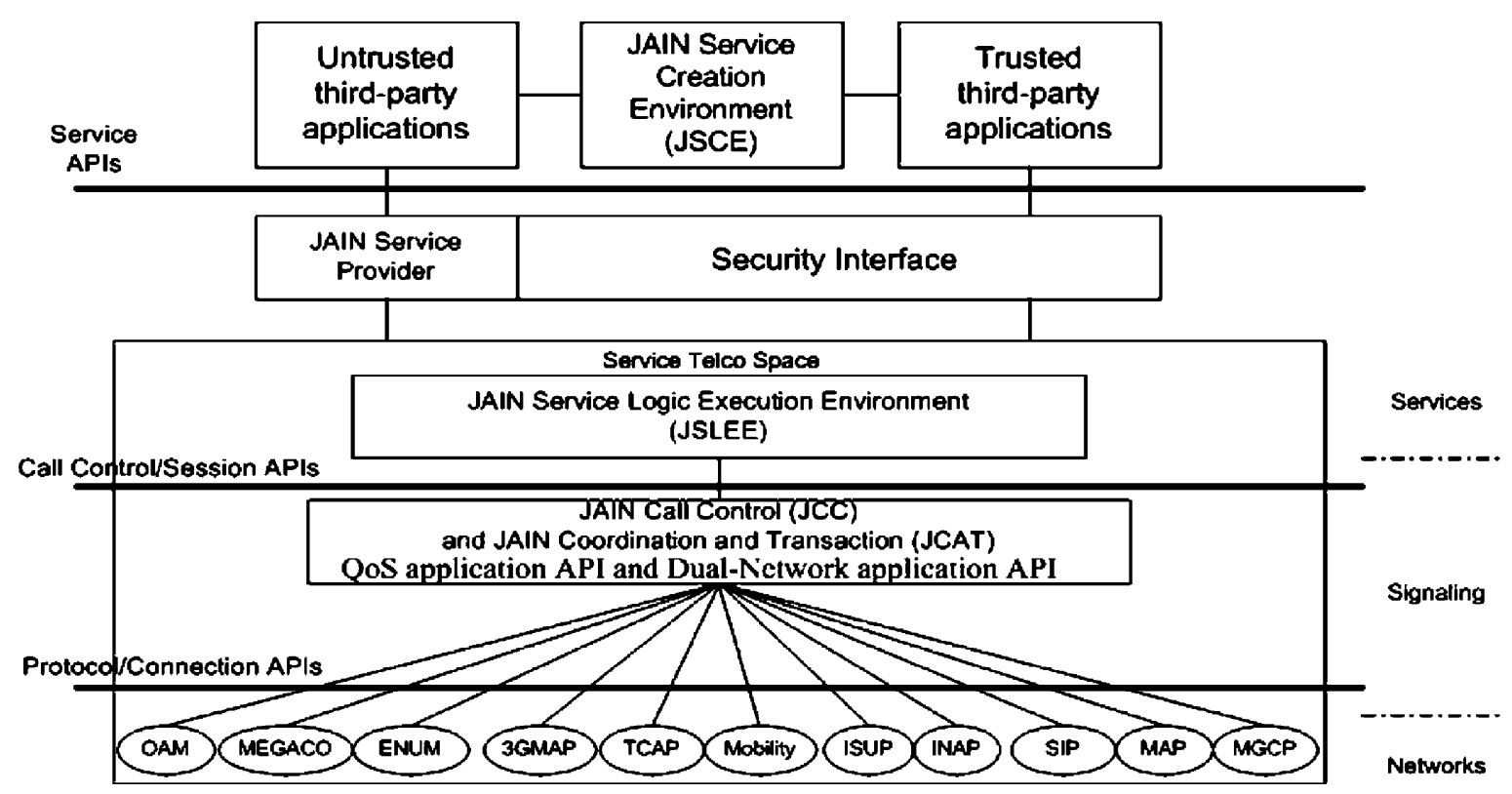

Fig. 12. JAIN API's structure.

APIs, and between the security and the data management and integration APIs. Therefore, the manufacturer can concentrate on developing applications for home networks. The OSGi standard is hoped to offer a bright future for all-IP network applications.

\section{Java APIs for Integrated Networks (JAIN) Platform}

The JAIN platform, developed by SUN, is based on JAVA technology [29]-[31]. The aim of the platform is to link the intelligent network to the Internet, to solve problems of compatibility among traditional intelligent networks. The platform includes a series of API's, which map and communicate with the hardware and telecommunication protocols of any network, making the applications portable. The standard interfaces allow the network applications to be developed rapidly on a Java platform in either wired or wireless environments. JAIN has three benefits: service portability, multinetwork integration, and network security support. Fig. 11 reveals that the JAIN platform has divided into four layers: the network layer, the protocol layer, the service layer, and the application layer.

JAIN APIs are grouped into two categories, protocol API and application API. The protocol API handles the concrete interface, including the network signaling protocol. The application API provides the functions required by upper-level services, and accesses the concrete protocol functions via the protocol API. The protocol API reflects the concrete protocol on the JAIN module. Fig. 12 reveals that the JAIN structure provides a service creation environment ( $\mathrm{SCE}$ ) to service trusted third-party applications in the interior network and the untrusted third-party applications in the outer network. These third-party applications can access the network's services via the secure service provider's interface. The JAIN platform also defined a series of development tools from the software component library, as well as the service logic execution environment (SLEE).

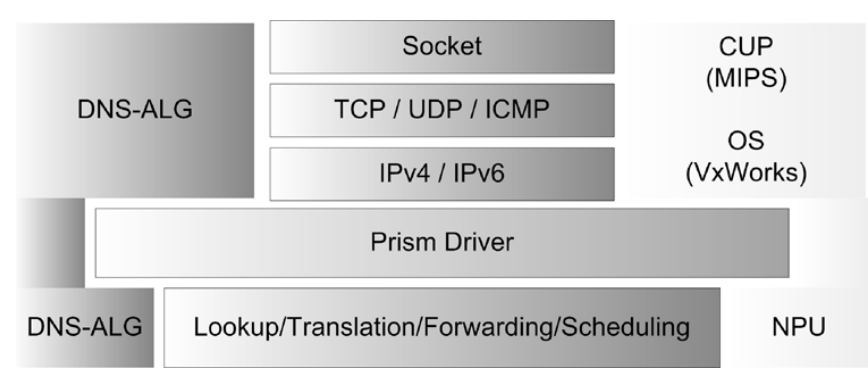

Fig. 13. System stack of NAT-PT on Vitesse IQ2000.

\section{SYSTEM IMPLEMENTATION}

This work emphasizes the application of the OSA-based platform in IPv4/IPv6 networks. The IPv4/IPv6 network architecture applied herein consists of an IPv4/IPv6 translator and a MR to support network mobility. The OSA-based platform was implemented to execute all-IPv6 network services with three applications: e-commerce, video-on-demand (VoD), and on-line games.

\section{A. IPv4/IPv6 Translation Based on Network Processor}

The main goal of the IPv4/IPv6 translator is to migrate networks seamlessly from IPv4 to IPv6. This work implemented the SIIT algorithm and the NAT-PT mechanism on the network processor. Fig. 13 depicts the system stack. The MIPS processor handles the control path, including the mapping table, the protocol stack and DNS-ALG. The NPU (FACET CPU) handles the data path, including table query, packet translation, packet delivery and packet scheduling. Additionally, some DNS-ALG functions, including the DNS message format conversion, are partially accomplished in the data path by NPU. Furthermore, the control path and data paths cooperate via a prism driver interface. 


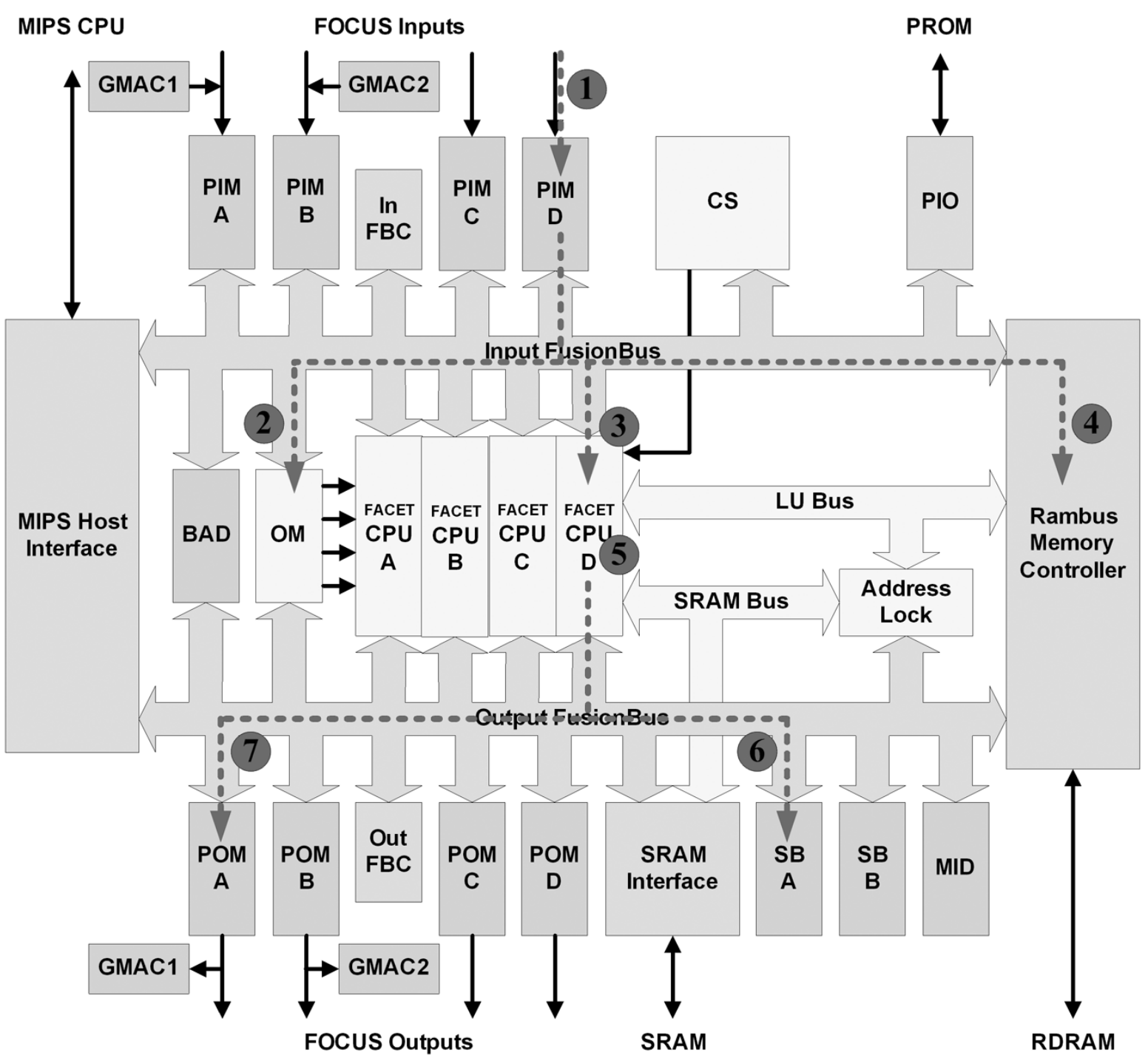

Fig. 14. Data processing flow chart on Vitesse IQ2000.

Fig. 14 depicts data processing in the network processor (Vitesse IQ2000), as described in the Vitesse IQ2000 Design Manual. Therefore, the implementation includes such fundamental functions as transmitting and receiving packets and initiating threads. Furthermore, Nonessential functions including packet translation and memory allocation were implemented. The complete system depends on the implemented functions.

\section{B. MR of Network Mobility}

Figs. 15 and 16 illustrate the NEMO testbed architecture for the network mobility MR. All machines of testbed are desktop PCs with an Intel CPU, 256 MB RAM and a 40 GB HDD. An 802.11b wireless card enabled connections between MR-HA and MR-AR. This work applied RedHat 9.0, including kernel 2.4.22, with the MIPL 1.0 patch. The MIPL source code was modified to incorporate the MN forwarding functions. A socket function was established to program the forwarding function, enable HA and MN to encapsulate and de-encapsulate packets

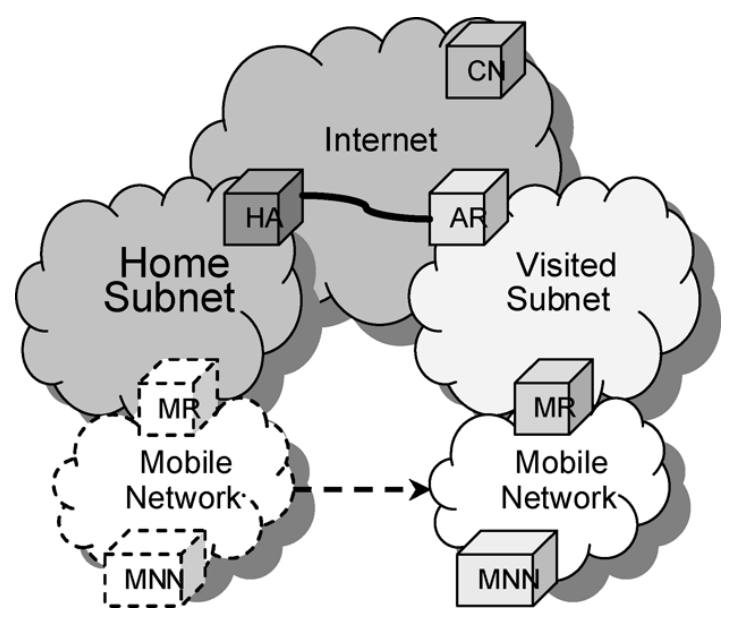

Fig. 15. NEMO simulation system.

and forward them to the proper networks, based on the routing table. 


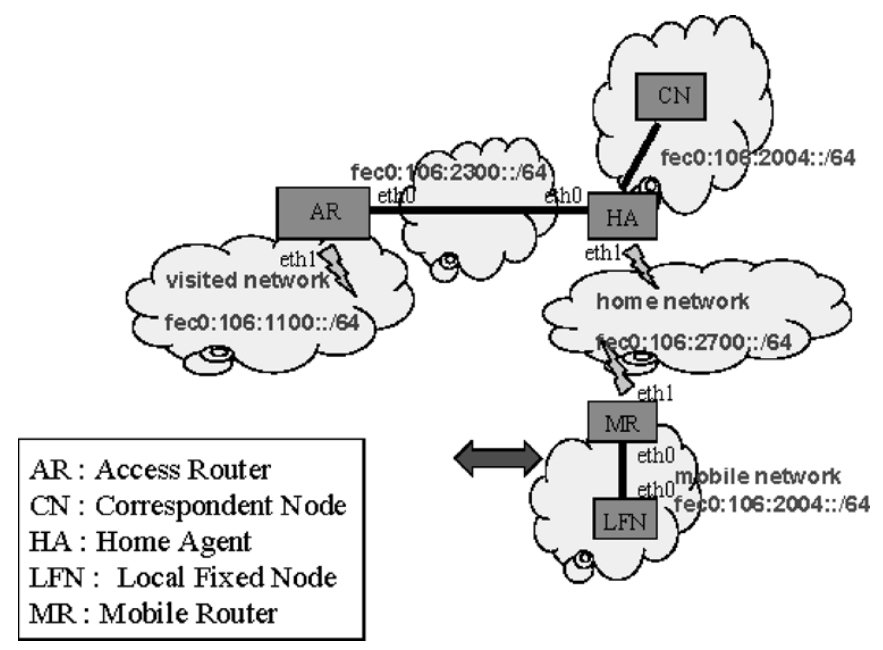

Fig. 16. NEMO architecture.

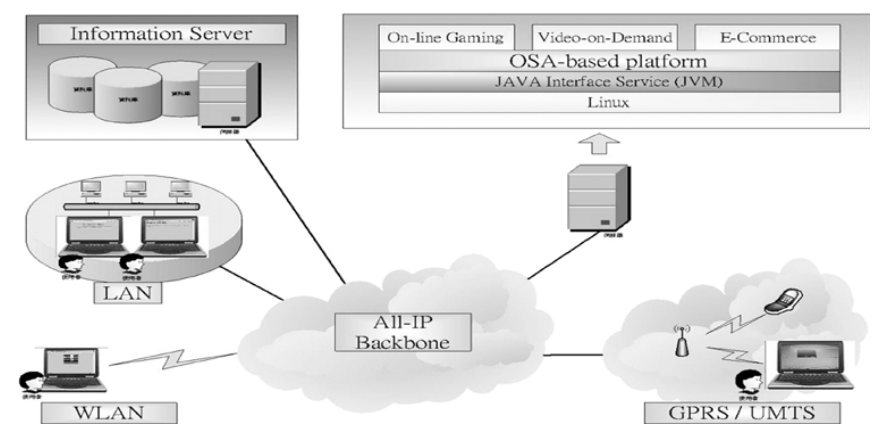

Fig. 17. Implementation environment.

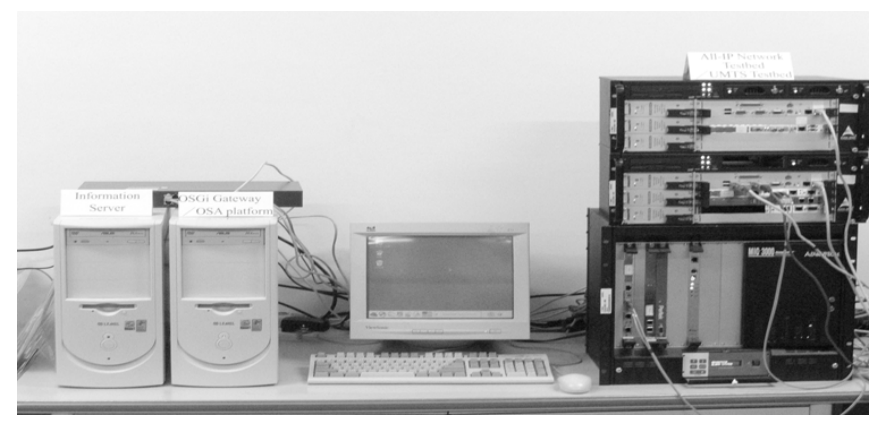

Fig. 18. All-IPv6 network environment with the OSA platform.

\section{OSA-Based Platform}

The prototype system implemented herein involves three main modules: server, communication, and client. Servers comprise the OSA-based platform and information server. The communication module is based on all-IPv6 networks. Clients include notebook computers and PDAs. Fig. 17 depicts the entire system architecture. Fig. 18 displays the all-IPv6 network environment and OSA platform. This network delivers applications through the $3 \mathrm{G}$ cellular network.

\section{Results AND PERFormance ANALYsis}

This work proposed an OSA-based application system was developed for all-IPv6 network services. The results and performance analysis in terms of qualitative and quantitative analyses were performed and described in the following.

\section{A. Developed Applications}

Three applications including e-commerce, $\mathrm{VoD}$, and on-line gaming were proposed to investigate the feasibility of the presented approach. Examples of these applications in various scenarios are given below.

1) E-Commerce Application: An increasing amount of trade is being conducted over networks, making the development of e-commerce applications very important. Two considerations of customers are security and convenience. The aim of this system is that it regards the bundle as its basic component. If programmers seek to add a new function, then they need only redevelop and reinstall a single bundle. They do not need to redevelop the entire system. For instance, a safety function can be added merely by introducing the certification and authorization bundle. A convenience function requires only the redevelopment of the bundle related to the user's mobility.

An on-line shopping scenario can be considered to elucidate the convenience of e-commerce. John registers with an Internet service provider on an OSA-based platform. One day he browses an on-line shopping website. He wants to buy an LCD screen, but he does not know which screen to buy. Therefore, he fills in a request form on the shopping website, and sends it to the website administrator. After several, the website administrator replies to John's message. After John's service company receives the message, it notifies John that they have received a message at his designated IP address. Then, when John looks at his PDA, he receives this message. He completes the order, and replies with details of the order. Then, his service company returns his message to the administrator of the on-line shopping website.

2) Video-on-Demand (VoD) Application: Fig. 19 depicts the scenario of a VoD application. First, the user links to the OSAbased platform from his office computer. The user then leaves the office. When he receives any messages away from home, he can set the OSA-based system to transmit messages to his mobile device automatically ("1" in Fig. 19). The user then wants to buy a movie ticket. He logs in into a recreational website, and inquires whether any movie tickets are available ("2" in Fig. 19). The recreational website receives this information and helps the user to may an enquiry to the movie company ("3" in Fig. 19). When the movie company sends a message, the website immediately sends it to the user, but the user is not in the office at this time. Accordingly, the message passes through the OSA-based platform to the user's mobile device ("4" and " 5 " in Fig. 19). After the user has received the message, the website notifies the movie company of its receipt. The movie company and the user can thus communicate with each other ("6" in Fig. 19). The user can select in advance the movie he wants to watch. Fig. 19 shows which movie was finally chosen.

3) On-Line Game Application: Mary is a student who takes the bus to school. She does not like waiting for the bus so she takes out a mobile device that can link to the Internet to find some video games to play. She finds a website that exclusively provides video games. This website is developed by using the OSA-based platform. Fig. 21 shows a frame of the website. Mary chooses one of the games, which is shown in Fig. 22. 


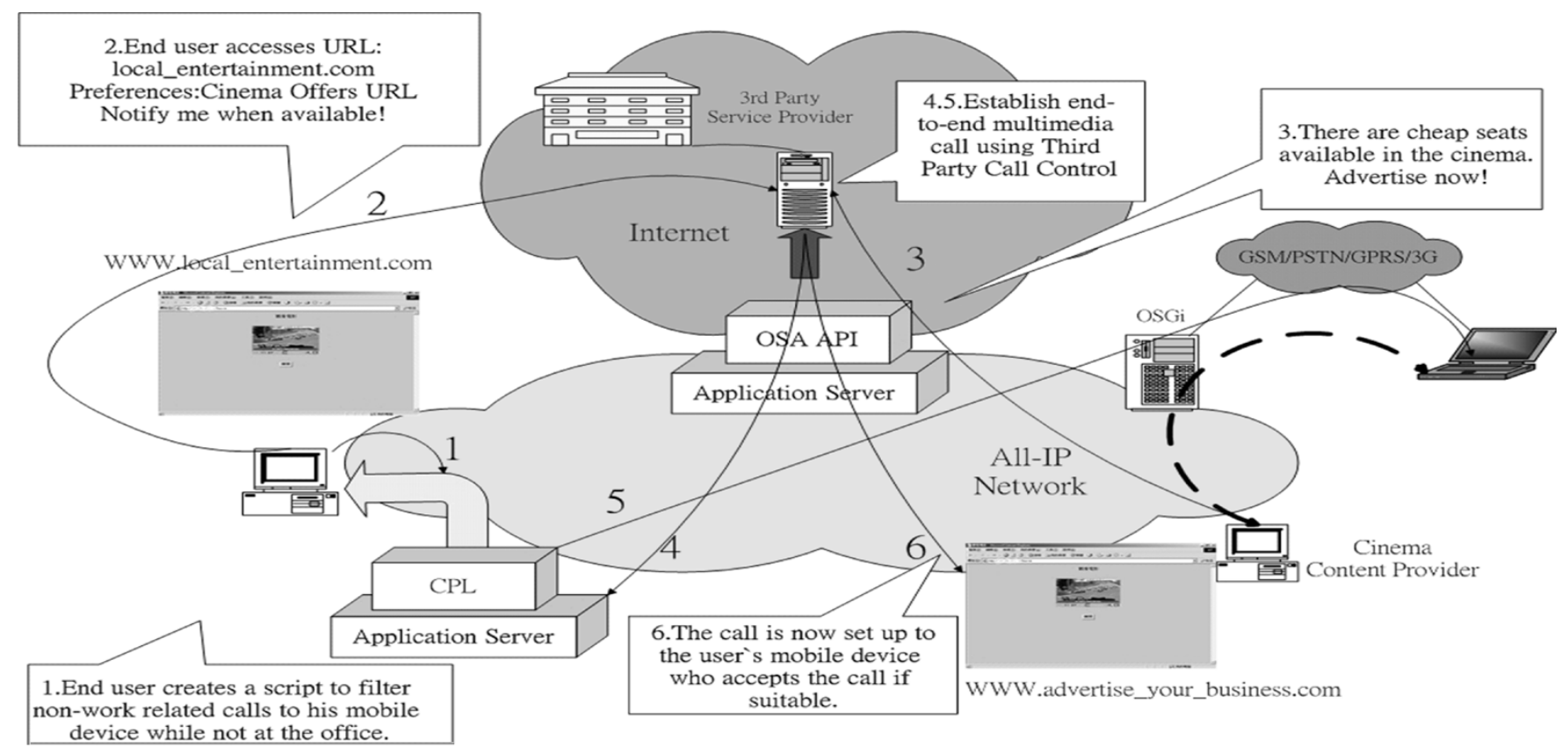

Fig. 19. VoD application scenario.

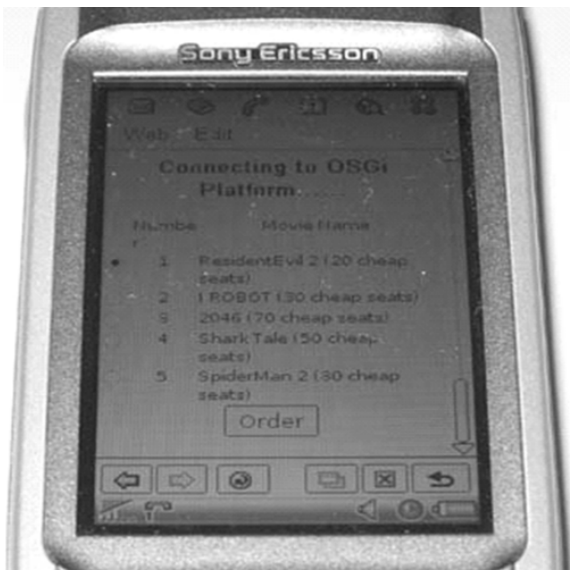

Fig. 20. VoD application.

\section{Welcome to the Game Player Center Connecting to OSGi Platform......}

\begin{tabular}{|c|c|ll|}
\hline \multicolumn{2}{|c|}{} & Number & \multicolumn{2}{c|}{ Game Name } \\
\hline C & 1 & Match & \\
\hline C & 2 & Rush & \\
\hline C & 3 & MoonStar & \\
\hline & & & OK \\
\hline
\end{tabular}

Fig. 21. On-line game application.

\section{B. Qualitative Analysis}

Table I presents a qualitative analysis. The value " 1 " refers to the best performance. The OSA-based system provides numerous advantages. It is a modular system, so has various features, including the reuse of programs, extensibility, flexibility,

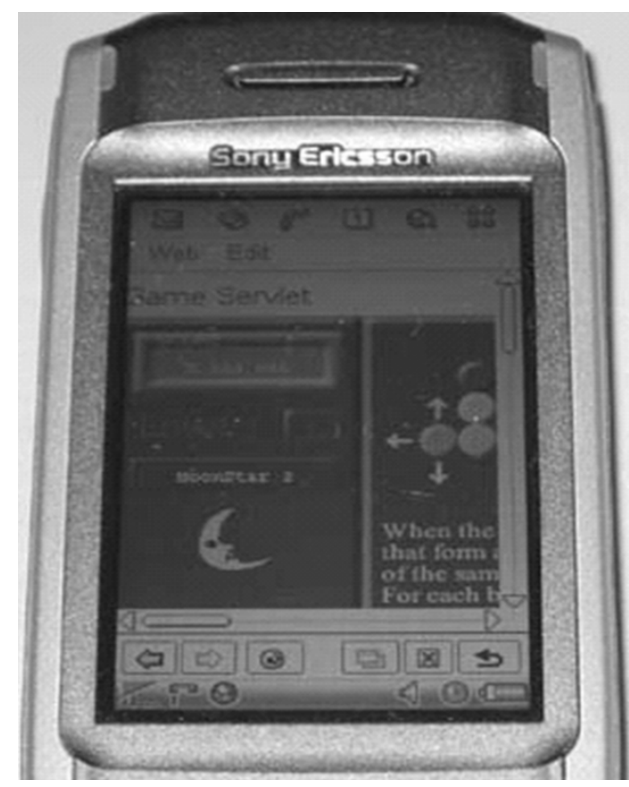

Fig. 22. Example of on-line game.

time of creation, and interoperability. A traditional networking system does not have such characteristics. Additionally, OSAbased systems with IPv6 networks have better mobility than those with IPv4 networks.

\section{Quantitative Analysis}

NetIQ's Chariot [32] can exactly simulate transaction traffic from real applications. This work selects NetIQ's Chariot as the simulation and analysis tool. NetIQ is a leading provider of systems management, security management, and web analytics cooperation. Chariot evaluates the performance of networked applications, performs stress tests on network devices 
TABLE I

ANALYSIS OF SYSTEM PERFORMANCE

\begin{tabular}{c|c|c|c}
\hline \hline Feature & $\begin{array}{c}\text { Traditional } \\
\text { System }\end{array}$ & $\begin{array}{c}\text { OSA-based System } \\
\text { OSA-based System with } \\
\text { IPv6 networks }\end{array}$ \\
\hline Reuse & 2 & 1 & 1 \\
\hline Extensible & 2 & 1 & 1 \\
\hline Creation Time & 2 & 1 & 1 \\
\hline Interoperability & 3 & 1 & 1 \\
\hline \hline
\end{tabular}

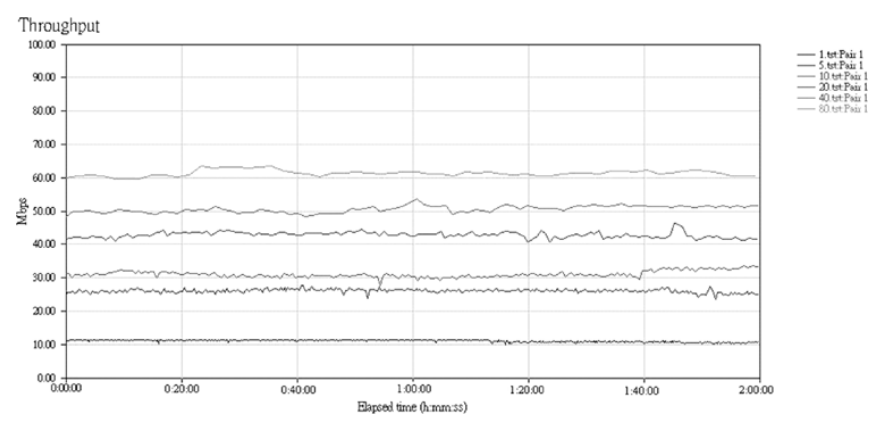

Fig. 23. Performance analysis—-system throughput.

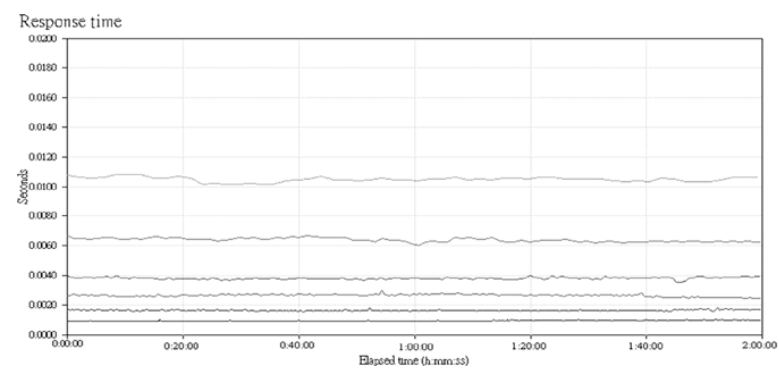

Fig. 24. Performance analysis_-average response time.

and predicts application performance prior to deployment. Additionally, we use Sniffer to measure and compute the application's delay time. Fig. 23 presents the system throughput of the OSA-based system. Data traffic of the same type is used to simulate the process by using a method that alters the data traffic with various users to measure the system throughput. The analytical data reveal that increasing the number of users increases the system throughput.

Fig. 24 presents the mean response time of the OSA-based system. The analytical data reveal that adding more users increases the response time. The response time was found to increase rapidly as the number of user increases above 80 . Therefore, the response time increases exponentially with the number of users until the system is out of service.

Fig. 25 presents the application delay time associated with various users. The service delay of a VoD application was evaluated using the Sniffer toolkit. The starting delay is higher than the average delay because of the random generating characteristics for VoD application. The delay time increases when a user connects to the OSA-based system. However, the delay

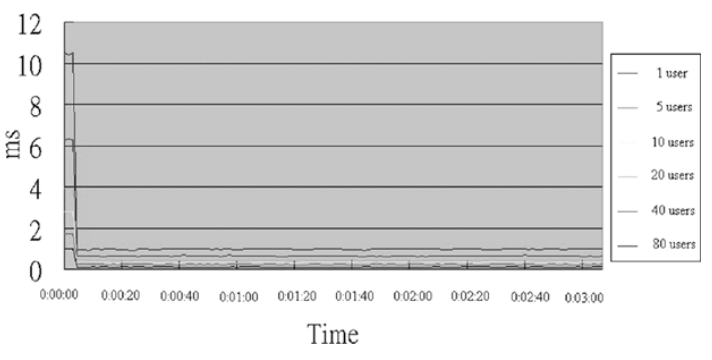

Fig. 25. Performance analysis—-delay time.

decreases when the user connects directly to the information server. Furthermore, adding users increases the delay time.

\section{CONCLUSION}

This work proposed an all-IPv6 service platform based on OSA to support a set of standard interfaces and applications. The all-IPv6 network environment was integrated with the OSA using an IPv4/IPv6 translator based on a network processor and an MR that supports IPv6 network mobility. The feasibility of the open service platform for all-IPv6 network environments and of the designed application programming interfaces was studied with reference to three applications: e-commerce, VoD, and on-line gaming. The performance analysis reveals that increasing the number of users from 1 to 80 increased the system throughput from 10.5 to $60.5 \mathrm{Mb} / \mathrm{s}$; the mean response time increased from approximately 1 to $10.5 \mathrm{~ms}$, and the delay increased from $0.1 \mathrm{~ms}$ to $1 \mathrm{~ms}$. We believe that all-IPv6 represent the likely future of the development of applied systems.

\section{REFERENCES}

[1] T. Dunn, "The IPv6 transition," IEEE Internet Comput., vol. 6, no. 3, pp. 11-13, May/Jun. 2002.

[2] H. Esaki, A. Kato, and J. Murai, "R\&D activities and testbed operation in WIDE project," in Proc. Symp. Applicat. Internet, Jan. 2003, pp. $172-177$.

[3] I. Raicu and S. Zeadally, "Impact of IPv6 on end-user applications," in Proc. 10th Int. Conf. Telecommun., vol. 2, Feb. 2003, pp. 973-980.

[4] "IPv6/IPv4 coexistence and migration," Microsoft, Washington, DC, White Paper, 2001.

[5] "IPv6-enabling the mobile Internet," Nokia, Finland, White Paper $10878,2000$.

[6] Nautilus6. [Online]. Available: http://www.nautilus6.org

[7] The IST OverDRiVE Project. [Online]. Available: http://www.ist-overdrive.org

[8] J. H. Kim, K. Leung, K. Echols, D. Shell, and M. Denny, "Demonstration of static network mobile router for mobile platforms," IEEE Creating the Information Force, vol. 2, pp. 746-750, Oct. 2001.

[9] T. Ernst, K. Uehara, and K. Mitsuya, "Network mobility from the InternetCAR perspective," in Proc. Int. Conf. Adv. Inf. Netw. Applicat., Mar. 2003, pp. 19-25.

[10] Uskela and Sami, "All IP architectures for cellular networks," in Proc. 3G Mobile Commun. Technol. Conf., Mar. 2001, pp. 180-185.

[11] "MPLS: The road to all-IP for wireless packet networks," Nortel Networks, White Paper, 2003.

[12] J. L. Chen, W. H. Chen, and S. Y. Kuo, "All-IPv6 service interworking gateway,” Int. J. Netw. Manage., pp. 135-147, Jan. 2005.

[13] L. Moretti and R. Depaoli, "OSA enabled global application roaming," in Proc. IEEE Intell. Netw. Workshop, 2001, pp. 135-139.

[14] E. Nordmark, "Stateless IP/ICMP translation algorithm (SIIT)," IETF, RFC 2765, 2000.

[15] G. Tsirtsis and P. Srisuresh, "Network address translation- protocol translation (NAT-PT)," IETF, RFC 2766, 2000.

[16] J. Manner and M. Kojo. (2004) Mobility related terminology. [Online]. Available: http://www.ietf.org/rfc/rfc3753.txt 
[17] D. Johnson, C. Perkins, and J. Arkko. (2004) Mobility support in IPv6. [Online]. Available: http://www.ietf.org/rfc/ rfc3775.txt

[18] D. Vijay, W. Ryuji, P. Alexandru, and T. Pascal. (2003) Network mobility (NEMO) basic support protocol. [Online]. Available: http://www.mobilenetworks.org/nemo/drafts/draft-ietf-nemo-basic-support-03.txt

[19] S. Hesham, C. Claude, E. Karim, and B. Ludovic. (2003) Hierarchical mobile IPv6 mobility management (HMIPv6). [Online]. Available: http://www.mobilenetworks.org/nemo/drafts/draft-ietf-mobileip-hmipv6-08.txt

[20] T. Ernst, A. Olivereau, L. Bellier, C. Castelluccia, and H.-Y. Lach. (2002) Mobile networks support in mobile IPv6 (prefix scope binding updates). [Online]. Available: http://www.mobilenetworks.org/ nemo/old/draft-ernst-mobileip-v6-network-03.txt

[21] A. Petrescu, M. Catalina-Gallego, C. Janneteau, H.-Y. Lach, and A. Olivereau. (2003) Issues in designing mobile IPv6 network mobility with the MR-HA bidirectional tunnel (MRHA). [Online]. Available: http://www. mobilenetworks.org/nemo/drafts/draft-petrescu-nemo-mrha-03.txt

[22] B. Odadzic and M. Jankovic, "Open service access (OSA) business models and service level agreement aspects," in Proc. IEEE Conf. Telecommun., vol. 1, Oct. 2003, pp. 22-25.

[23] W. Wu and H. Zou, "Design OSA/Parlay application frameworks using a pattern language," in Proc. IEEE Conf. Commun. Technol., vol. 2, Apr. 2003, pp. $1558-1561$.

[24] A. J. Moerdijk and L. Klostermann, "Opening the networks with Parlay/OSA: Standards and aspects behind the APIs," IEEE Netw., vol. 17, no. 3, pp. 58-64, May-Jun. 2003.

[25] D. Marples and P. Kriens, "The open service gateway initiative-An introductory overview," IEEE Commun. Mag., vol. 39, no. 12, pp. 110-114, Dec. 2001

[26] K. C. Kang and J. W. Lee, "Implementation of management agents for an OSGi-based residential gateway," in Proc. IEEE Conf. Commun. Technol., vol. 2, Feb. 2004, pp. 1103-1107.

[27] R. S. Hall and H. Cervantes, "An OSGi implementation and experience report," in Proc. IEEE Conf. Consum. Commun. Netw., Jan. 2004, pp. 394-399.

[28] C. Lee, D. Nordstedt, and S. Helal, "Enabling smart spaces with OSGi," IEEE Pervasive Comput., vol. 2, no. 3, pp. 89-94, 2003.

[29] (2002) The JAIN APIs: Integrated Network API's for the JAVA Platform. White Paper. [Online]. Available: http://java.sun.com/products/jain/

[30] D. Tait, J. Keijzer, and R. Goedman, "JAIN: A new approach to services in communication networks," IEEE Commun. Mag., vol. 38, no. 1, pp. 94-99, Jan. 2000.

[31] R. Jain, F. M. Anjum, P. Missier, and S. Shastry, "Java call control, coordination, and transactions," IEEE Commun. Mag., vol. 38, no. 1, pp. 108-114, 2000.

[32] NetIQ. [Online]. Available: http://www.netiq.com/

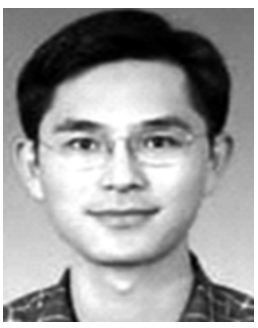

Yao-Chung Chang is working towards the Ph.D. degree in the Department of Computer Science and Information Engineering, National Dong Hwa University (NDHU), Hualien, Taiwan, R.O.C.

$\mathrm{He}$ is a member of the R\&D Division, Taiwan National Information and Communications Initiative (NICI) IPv6 Steering Committee. Currently, he is working on the Mobile IPv6 API for Win CE Project of the R\&D Division. His main research focuses on the network related topics including transition of IPv4/IPv6, Mobile IPv6, network mobility, and

sensor network

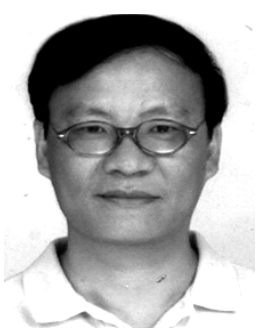

Jiann-Liang Chen was born in Taiwan on December 15, 1963. He received the Ph.D. degree in electrical engineering from the National Taiwan University, Taipei, Taiwan, R.O.C., in 1989.

Since August 1997, he has been with the Department of Computer Science and Information Engineering, National Dong Hwa University (NDHU), Hualien, Taiwan, R.O.C., where he is a Professor. His current research interests are directed at cellular mobility management and personal communication systems

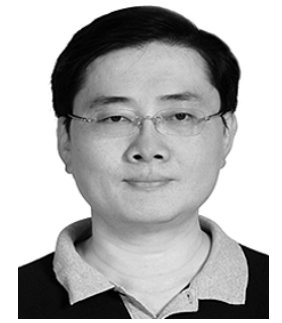

Han-Chieh Chao (SM'04) received the M.S. and $\mathrm{Ph} . \mathrm{D}$. degrees in electrical engineering from Purdue University, West Lafayette, IN, in 1989 and 1993, respectively.

$\mathrm{He}$ is a Full Professor in the Department of Electronic Engineering and Dean of the Library and Information Technology Office, National Ilan University, I-Lan, Taiwan, R.O.C. He has had 34 M.S.E.E. thesis students. He also received many funded research grants from the NSC, the Ministry of Education (MOE), RDEC, the Industrial Technology of Research Institute, the Institute of Information Industry, and the FarEasTone Telecommunications Laboratory. He has been invited frequently to give talks at national and international conferences and research organizations. He is Executive Editor of the Journal of Internet Technology and the Editor-in-Chief for the International Journal of Internet Protocol Technology and the International Journal of Ad Hoc and Ubiquitous Computing. He has authored or coauthored four books and has published about 140 refereed professional research papers. His research interests include high-speed networks, wireless networks, IPv6-based networks, digital creative arts, and digital divide.

Dr. Chao has received many research awards, including Purdue University SRC Awards, and the NSC Research Awards (National Science Council of Taiwan). He is also serving as an IPv6 Steering Committee Member and Co-Chair of R\&D Division of the National Information and Communication Initiative (NICI), a ministry level government agency which aims to integrate domestic IT and Telecom projects of Taiwan), and Co-Chair of the Technical Area for IPv6 Forum Taiwan.

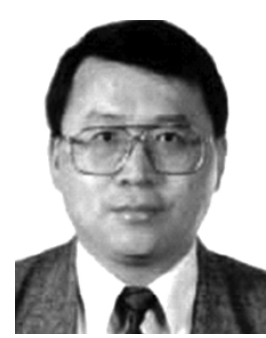

Sy-Yen Kuo (M'88-SM'98-F'01) received the B.S degree in electrical engineering from the National Taiwan University, Taipei, Taiwan, R.O.C., in 1979, the M.S. degree in electrical and computer engineering from the University of California, Santa Barbara, in 1982, and the Ph.D. degree in computer science from the University of Illinois at Urbana-Champaign, Urbana, in 1987.

$\mathrm{He}$ is a Professor at the Department of Electrical Engineering, National Taiwan University and was the Chairman from 2001 to 2004. Currently, he is on sabbatical leave and serving as a Visiting Professor at the Computer Science and Engineering Department, the Chinese University of Hong Kong. He spent his sabbatical year as a Visiting Researcher at AT\&T Laboratories-Research, NJ, from 1999 to 2000. He was the Chairman of the Department of Computer Science and Information Engineering, National Dong Hwa University, Hualien, Taiwan, R.O.C., from 1995 to 1998, a faculty member in the Department of Electrical and Computer Engineering, University of Arizona from 1988 to 1991, and an Engineer at Fairchild Semiconductor and Silvar-Lisco, both in California, from 1982 to 1984. In 1989, he also worked as a Summer Faculty Fellow at the Jet Propulsion Laboratory, California Institute of Technology. He has published more than 180 papers in journals and conferences. His current research interests include dependable systems and networks, software reliability engineering, mobile computing, and reliable sensor networks.

Prof. Kuo is a member of the IEEE Computer Society. He received the Distinguished Research Award (1997-2005) from the National Science Council, Taiwan. He was also a recipient of the Best Paper Award in the 1996 International Symposium on Software Reliability Engineering, the Best Paper Award in the simulation and test category at the 1986 IEEE/ACM Design Automation Conference (DAC), the National Science Foundation's Research Initiation Award in 1989, and the IEEE/ACM Design Automation Scholarship in 1990 and 1991. 